\title{
Index rerum ad Vol. 162
}

\section{Confecit: C. Loeb-Schärch}

$(B)=$ Buchbesprechungen - Book reviews - Livres nouveaux $(V)=$ Vortrag - Report Communication

Ablatio, v. Retinal detachment

ABO blood groups and the secretor status in patients with uveitis, 298

Acuité visuelle, v. Acuity testing, Amyloidosis, Ectopia lentis, Netz-hautgefässschattenfigur,

Skiagram

Acuity testing; do we really wish standardization of acuity testing?, 235 (V)

Aderhaut, v. Chorioretinal inflammatory lesions, Choroidal fluorescence, Inflammations uvéales, Lightcoagulation, Transscleral freezing

Amyloidosis; unusual cause of vitreous opacities. Primary familial amyloidosis, 173

Anatomie, v. Amyloidosis, Hydran-encephaly, Listeriosis

Anencephaly, v. Hydranencephaly

Angiitis, v. Wegenersche Granulo-matose

Angiographie, v. Calcium dobesilate, Chorioretinal inflammatory lesions, Choroidal

fluorescence, Gefässverschlüsse, Leuko-encephalitis, Retinitis pigmentosa

Angioscotomata and the choroidal pattern, 281 (V)

Antigen-Antikörper-Reaktion, v. Wegenersche Granulomatose

Applanations-Tonometer; zur Des-infektion der Messkörperchen des Applanations-Tonometers nach Goldmann, 129

Aqueous humor, v. Cholinesterase

Augenbinnendruck, v. Applanations-Tonometer, Follow-up, Gefässverschlüsse, Glaucoma

Augenkomplikationen, v. Candida albicans, Ectopia lentis, Strumek-tomie

Augenmuskeln, v. Ophthalmoplegie, Parkinson's disease, Trochlear paralysis, Vertikale

Blicklähmung

Bakteriologie, v. Applanations-Tonometer, Candida albicans, Listeriosis

Bardet-Biedl; investigations cliniques et génétiques sur le syndrome de Bardet-Biedl en Suisse, 318 (B)

BelГsches Phänomen, v. Vertikale Blicklähmung

Bindehaut, v. Extreme strabismus, Malignant melanoma, Wegenersche Granulomatose

Binokularsehen, v. Subliminal stimuli

Blickfeld, v. Schieloperationen

Blicklähmung, v. Ophthalmoplegie, Vertikale Blicklähmung

Blood group, v. ABO blood groups

Blutgruppe, v. ABO blood groups

Blutung, v. Newborn

Bogaert, v. Leukoencephalitis

Book reviews, 318 (B)

Buchbesprechungen, 318 (B) 
Calcium dobesilate; the action of calcium dobesilate on capillary permeability in diabetics, 33

Candida albicans; Candida albicans-Endophthalmitis nach Katarakt-extraktion, 160

Capillary permeability, v. Calcium dobesilate

Case reports; clinicopathologic case reports, 151-192

Caterpillar, v. Raupenhaare

Central nervous system, v. Hydranencephaly, Leukoencephalitis, Ophthalmoplegie, Parkinson's disease, Vertikale Blicklähmung

Index rerum

ad Vol. 162

369

Cerebrum, v. Hydranencephaly, Leukoencephalitis, Ophthalmo-plegie, Parkinson's disease, Vertikale Blicklähmung

Champ du regard, v. Schiel-operationen

Champ visuel, v. Angioscotomata, Follow-up, Netzhautgefäss-schattenfigur, Retinitis

pigmentosa

Chenille, v. Raupenhaare

Chirurgie, v. Duane's retraction syndrome, Ectopia lentis, Extreme strabismus, Pseudophakoi, Schiel-operationen, Schmalmesserschnitt, Stereoscopic image amplifier Strumektomie,

Zerreissfestigkeit

Chitin, v. Raupenhaare

Cholinesterase in aqueous humor of man and open-angle glaucoma, 60

Chorioidea, v. Chorioreíinal

inflammatory lesions, Choroidal fluorescence, Inflammations uvéales, Lightcoagulation, Transscleral freezing

Chorioretinal inflammatory lesions; fluorescein-angiographical patterns of chorioretinal

inflammatory lesions, $261(\mathrm{~V})$

Chorioretinitis, v. ABO blood groups, Chorioretinal inflammatory lesions, Inflammations

uvéales

Choroidal fluorescence in the normal human eye, 246 (V)

Circulation, v. Gefässverschlüsse

Clinicopathologic case reports, 151-192

Coagulation à la lumière, v. Light-coagulation, Retinitis centralis serosa

Complamine, v. Vascular occlusion

Complications oculaires, v. Candida albicans, Ectopia lentis, Strumektomie

Computer; planning for computer processing of records in an ophthalmology outpatient clinic,

21

Congrès, v. Netherlands Ophthalmo-logical Society

Conjunctiva, v. Extreme strabismus, Malignant melanoma, Wegener-sche Granulomatose

Cornea, v. Raupenhaare, Wegener-sche Granulomatose

Corps étranger, v. Raupenhaare, Stereoscopic image amplifier

Corps vitré, v. Amyloidosis, Retinal detachment, Transscleral freezing, Vitreous cells

Cristallin, v. Ectopia lentis, Pseudophakoi

Déchirure scléraíe, v. Zerreissfestigkeit

Décollement rétinien, v. Retinal detachment

Dégénérescence maculaire, v. Laurence-Moon-Bardet-Biedl syndrome 
Dégénérescence tapéto-rétinienne, v. Laurence-Moon-Bardet-Biedl syndrome, Retinal detachment, Retinitis pigmentosa

Desinfektion, v. Applanations-Tonometer

Diabetes, v. Calcium dobesilate

Diagnostik, v. Glaucoma, Inflammations uvéales, Trochlear paralysis, Vertikale Blicklähmung

Doxium, v. Calcium dobesilate

Duane's retraction syndrome; the surgical treatment of Duane's retraction syndrome, 284 (V)

Ectopia lentis; the results of my operations improving visual acuity of ectopia lentis, 98

Elektrokoagulation, v. Zerreissfestigkeit Electromyogram, v. Parkinson's

disease Electrooculogram, v. Retinitis

pigmentosa Electroretinogram, v. Macular ERG,

Retinitis pigmentosa EMG, v. Parkinson's disease Endokrinologie, v. Strumektomie

Endophthalmitis, v. Candida albicans Entoptic visual functions, v. Netz-

hautgefässschattenfigur Entzündung, v. ABO blood groups, Chorioretinal inflammatory lesions, Inflammations uvéales, Retinitis centralis serosa EOG, v. Retinitis pigmentosa Epithelium

pigmentaire, v. Choroidal fluorescence, Leukoencephalitis

370

Index rerum

ad Vol. 162

ERG, v. Macular ERG, Retinitis pigmentosa

Excavation papillaire, v. Angioscotomata, Glaucoma

Exophthalmus, v. Strumektomie

Experimentelle Forschung, v. Cholin-esterase, Subliminal stimuli, Transscleral freezing,

Zerreiss-festigkeit

Extreme strabismus; surgical correction of extreme strabismus, 244 (V)

Fasciculus longitudinalis medialis, v. Ophthalmoplegie

Favre's vitreo-tapeto-retinal degeneration, v. Retinal detachment

Field of fixation, v. Schiel-operationen

Fixation, v. Heterophoria

Fluorescein-Angiographie, v.

Calcium dobesilate, Chorioretinal inflammatory lesions, Choroidal fluorescence,

Gefässverschlüsse, Leukoencephalitis, Retinitis pigmentosa

Follow-up; a five-year follow-up of subjects with intra-ocular pressure of $22-30 \mathrm{~mm} \mathrm{Hg}$ without anomalies of optic nerve and visual field typical for glaucoma at first investigation, 288 (V)

Fonctions visuelles entoptiques, v. Netzhautgefässschattenfigur

Foreign body, v. Raupenhaare, Stereoscopic image amplifier

Fovea, v. Leukoencephalitis, Macular ERG

Foveal differential treshold, v. Subliminal stimuli

Fremdkörper, v. Raupenhaare, Stereoscopic image amplifier

Fusion, v. Heterophoria

y-System, v. Parkinson's disease

Geburtshilfe, v. Newborn

Gefässe, v. Angioscotomata, Calcium dobesilate, Chorioretinal inflammatory lesions, Choroidal

fluorescence, Gefässverschlüsse, Leukoencephalitis, Netzhautgefässschattenfigur, Newborn, Vascular occlusion 
Gefässverschlüsse; zur Klinik und Therapie der akuten Gefässverschlüsse, 65 (V)

Genetics, v. Amyloidosis, Ectopia lentis, Retinal detachment, Retinitis pigmentosa

Germasept, v. Applanations-Tono-meter

Gesichtsfeld, v. Angioscotomata, Follow-up, Netzhautgefässschattenfigur, Retinitis pigmentosa

Glaskörper, v. Amyloidosis, Retinal detachment, Transscleral freezing, Vitreous cells

Glaucoma, v. Angioscotomata, Cholinesterase, Follow-up, Vitreous cells

Glaucoma; the early diagnosis of glaucoma, 276 (V)

Granulomatose, v. Wegenersche Granulomatose

Groupe sanguin, v. ABO blood groups

Haemorrhagie, v. Newborn Hemoglobinopathy, v. Retinal

detachment Heparin, v. Vascular occlusion Hérédité, v. Amyloidosis, Ectopia lentis, Retinal detachment, Retinitis pigmentosa Heterophoria; a new theory about

heterophoria, 199 (V) Histologie, v. Amyloidosis, Candida albicans, Hydranencephaly,

Laurence-Moon-Bardet-Biedl syndrome, Leukoencephalitis, Lightcoagulation, Listeriosis,

Lymphoma, Malignant melanoma, Retinal detachment, Transscleral freezing, Vitreous cells,

Wegenersche Granulomatose Hornhaut, v. Raupenhaare,

Wegenersche Granulomatose Humeu $\Gamma$ aqueuse, v. Cholinesterase Hydranencephaly; eye

findings in hydranencephaly, $151(\mathrm{~V})$

Inflammation, v. ABO blood groups, Chorioretinal inflammatory lesions, Inflammations uvéales,

Retinitis centralis serosa

Index renim

ad Vol. 162

371

Inflammations uvéales; les inflammations uvéales. Reflexions après 20 ans d'observation sanatoriale, 11

Instrument, v. Stereoscopic image amplifier

Intraocular pressure, v. Applana-tions-Tonometer, Follow-up, Gefässverschlüsse, Glaucoma

Iris, v. Pseudophakoi

Kammerwasser, v. Cholinesterase

Kammerwasserpunktion, v. Inflammations uvéales

Kaninchen, v. Transscleral freezing, Zerreissfestigkeit

Kapillarpermeabilität, v. Calcium dobesilate

Katarakt, v. Candida albicans, Schmalmesserschnitt

Konvergenzlähmung, v. Vertikale Blicklähmung

Kreislauf, v. Gefässverschlüsse

Kryothermie, v. Transscleral freezing, Zerreissfestigkeit

Lähmung, v. Ophthalmoplegie, Trochlear paralysis, Vertikale Blicklähmung

Lapin, v. Transscleral freezing, Zerreissfestigkeit

Laurence-Moon-Bardel-Biedl syndrome with juvenile macular degeneration. Clinical and pathological study, 76

Lens, v. Ectopia lentis, Pseudophakoi

Leuchtreize, v. Subliminal stimuli

Leukoencephalitis; fluoroangio-graphic picture of the macular lesion in subacute sclerosing leukoencephalitis van Bogaert, 348

Lichtkoagulation, v. Light-coagulation, Retinitis centralis serosa 
Lid, v. Lymphoma

Lightcoagulation; recurrence of malignant melanoma of the choroid seven and eight years after lightcoagulation, 188

Linse, v. Ectopia lentis, Pseudophakoi

Listeriosis; congenital listeriosis with ocular involvement, 167

Livres nouveaux, 318 (B) Lymphoma; malignant lymphoma of lid margin, 178

Macula, v. Leukoencephalitis, Macular ERG

Maculadegeneration, v. Laurence-Moon-Bardet-Biedl syndrome

Macular ERG; the combined use of the macular electroretinogram (M-ERG) and the visually

evoked responses (VER), 208 (V)

Malformation, v. Hydranencephaly

Malignant melanoma of palpebral conjunctiva, 183

Medikament, v. Applanations-Tonometer, Calcium dobesilate, Vascular occlusion

Melanoma, v. Lightcoagulation, Malignant melanoma

Methode, v. Computer, Schiel-

operationen, Schmalmesserschnitt, Skiagram

Missbildung, v. Hydranencephaly

Morphology; the retina morphology, 318 (B)

Motilität, v. Ophthalmoplegie, Schiel-operationen, Vertikale Blicklähmung

Multiple Sklerose, v. Ophthalmoplegie

Muscles oculaires, v. Ophthalmoplegie, Parkinson's disease, Trochlear paralysis, Vertikale

Blicklähmung

Mykose, v. Candida albicans

Myopia progressiva, v. Retinal detachment

Neovascularisation, v. Calcium dobesilate

Netherlands Ophthalmological Society. 161st meeting, Leiden 1968, 194-297 (V)

Netzhaut, v. Gefässverschlüsse, Laurence-Moon-Bardet-Biedl syndrome, Leukoencephalitis, Macular ERG, Netzhautgefäss-schattenfigur, Newborn, Retinal detachment, Retinitis centralis serosa, Retinitis pigmentosa, Subliminal stimuli, Transscleral freezing

372

Index rerum

ad Vol. 162

Netzhautgefässschattenfigur; welche Gesichtsfeldbereiche kann man mit der

Netzhautgefässschattenfigur erfassen?, 140

Neuro-Ophthalmologie, v. Ophthalmoplegie, Vertikale Blicklähmung

Newborn; retinal haemorrhage in the newborn. An attempt to indicate and explain its cause and significance, $213(\mathrm{~V})$

Norries disease, v. Retinal detachment

Nystagmus, v. Ophthalmoplegie

Nystagmus retractorius, v. Vertikale Blicklähmung

Obstetrics, v. Newborn

Operation, v. Duane's retraction syndrome, Ectopia lentis, Extreme strabismus, Pseudophakoi,

Schiel-operationen, Schmalmesserschnitt, Stereoscopic image amplifier, StГumektomie,

Zerreissfestigkeit

Ophthalmodynamometrie, v. Gefäss-verschlüsse 
Ophthalmoplegie; die internukleare Ophthalmoplegie, 331

Paedo-Ophthalmologie, v. Newborn

Palsy, v. Ophthalmoplegie, Trochlear paralysis, Vertikale Blicklähmung

Papillarexkavation, v. Angio-scotomata, Glaucoma

Paralysie, v. Ophthalmoplegie, Trochlear paralysis, Vertikale Blicklähmung

Parinaud'sche Krankheit, v. Vertikale Blicklähmung

Parkinson's disease; impairment of function of the extraocular muscles in Parkingson's disease, 343

Periarteriitis nodosa, v. Wegener-sche Granulomatose

Peripheral vision, v. Skiagram

Perméabilité capillaire, v. Calcium dobesilate

Photokoagulation, v. Light-coagulation, Retinitis centralis serosa

Pigmentepithel, v. Choroidal fluorescence, Leukoencephalitis

Ponction camérulaire, v. Inflammations uvéales

Pression oculaire, v. Applanations-

Tonometer, Follow-up, Gefäss-

verschlüsse, Glaucoma Proceedings, v. Netherlands

Ophthalmological Society Pruning, v. Acuity testing Pseudophakoi; the attachment of pseudophakoi, 205 (V) Ptosis, v. Ophthalmoplegie Pupille, v. Vertikale Blicklähmung

Rabbit, v. Transscleral freezing, Zerreissfestigkeit

Raupenhaare; über Augenschädi-gungen durch Raupenhaare, 308

Recherches expérimentales, $v$.

Cholinesterase, Subliminal stimuli, Transscleral freezing, Zerreissfestigkeit

Refraktion, v. Skiagram

Retina, v. Gefässverschlüsse, Laurence-Moon-Bardet-Biedl syndrome, Leukoencephalitis,

Macular ERG, Netzhautgefässschattenfigur, Newborn, Retinal detachment, Retinitis centralis serosa, Retinitis pigmentosa, Subliminal stimuli, Transscleral freezing

Retinal correspondence, v. Heíero-phoria

Retinal detachment; paihoiogy of hereditary conditions related to retinal detachment, 223 (V)

Retinitis centralis serosa, 287 (V)

Retinitis pigmentosa; on the patho-genesis of retinitis pigmentosa, 82

Retinoschisis hereditaria juvenilis, v. Retinal detachment

Retinoscopy, v. Skiagram

Retraktion des Oberlids, v. Vertikale Blicklähmung

Rheomacrodex, v. Vascular occlusion

Röntgen, v. Stereoscopic image amplifier

Sclérose en plaques, v. Ophthalmoplegie

Scotoma, v. Angioscotomata, Macular ERG

Schielen, v. Extreme strabismus, Heterophoria, Schieloperationen

Index $\Gamma$ erum

ad Vol. 162

373

Schieloperationen; Motilitäts verhal-ten in Abhängigkeit von verschie-denen Schieloperationen, 115

Schmalmesserschnitt und Stufen-schnitt bei der Kataraktoperation, 355 
Schwelle fovealer Differenzierung, v. Subliminal stimuli

Secretor status, v. ABO blood groups

Sehschärfe, v. Acuity testing, Amy-loidosis, Ectopia lentis, Netzhaut-gefässschattenfigur,

Skiagram

Seuil différentiel fovéal, v. Subliminal stimuli

Skiagram; peripheral retinoscopy and the skiagram, 1

Skiaskopie, v. Skiagram

Sklera, v. Transscleral freezing. Wegenersche Granulomatose, Zerreissfestigkeit

Squint, v. Extreme strabismus, Heterophoria, Schieloperationen

Standardization, v. Acuity testing

Stargardt'sche Maculadegeneration, v. Laurence-Moon-Bardet-Biedl syndrome

Stereoscopic image amplifier for the removal of intra-ocular and extra-ocular non-magnetic

foreign bodies, $273(\mathrm{~V})$

Stimulation lumineuse, v. Subliminal stimuli

Strabismus, v. Extreme strabismus, Heterophoria, Schieloperationen

Strumektomie; die iatrogenen Gefahren der Strumektomie mit besonderer Rücksicht auf die

ophthalmologischen Beziehungen, Ill

Stufenschnitt, v. Schmalmesserschnitt

Subliminal stimuli; monocular and binocular responses to liminal and subliminal stimuli, 41

Surgery, v. Duane's retraction

syndrome, Ectopia lentis, Extreme strabismus, Pseudophakoi, Schieloperationen,

Schmalmesserschnitt, Stereoscopic image amplifier, Strumektomie, Zerreissfestigkeit

Syndrome, v. Duane's retraction syndrome, Laurence-Moon-Bardet-Biedl syndrome

Système nerveux central, v. Hydran-encephaly, Leukoencephalitis, Ophthalmoplegie,

Parkinson's disease, Vertikale Blicklähmung

Tapetoretinale Degeneration, v. Laurence-Moon-Bardet-Biedl syndrome, Retinal detachment, Retinitis pigmentosa

Tego 103 S, v. Applanations-Tonometer

Tension oculaire, v. Applanations-Tonometer, Follow-up, Gefäss-verschlüsse, Glaucoma

Test, v. Acuity testing

Therapie, v. Calcium dobesilate, Duane's retraction syndrome, Ectopia lentis, Extreme

strabismus, Gefässverschlüsse, Lympho-ma, Malignant melanoma, Pseudophakoi, Raupenhaare,

Vascular occlusion, Wegenersche Granulomatose

Thyreoidektomie, v. Strumektomie

Tonometer, v. Applanations-Tonometer

Toxoplasmose, v. Inflammations uvéales

Transscleral freezing; experimental transscleral freezing at temperatures ranging from -20 to $160^{\circ} \mathrm{C}, 194(\mathrm{~V})$

Trochlear paralysis; missed diagnosis of a case of trochlear paralysis, 242 (V)

Tuberkulose, v. Inflammations uvéales

Tumor, v. Lightcoaguiation,

Lymphoma, Malignant melanoma

Umblickfeld, v. Schieloperationen Uveitis, v. ABO blood groups, Inflammations uvéales Vaisseaux, v. Angioscotomata, Calcium dobesilate, Chorioretinal inflammatory lesions, Choroidal fluorescence, Gefässverschlüsse, Leukoencephalitis, Netzhautgefäss-schattenfigur, Newborn, Vascular occlusion 
Van Bogaert, v. Leukoencephalitis

Varia, 128, 319

374

Index rerum

ad Vol. 162

Vascular occlusion; the treatment of impending vascular occlusion with Rheomacrodex, Heparin and Complamine, 296 (V)

VER, v. Macular ERG

Vererbung, v. Amyloidosis, Ectopia lentis, Retinal detachment, Retinitis pigmentosa

Verhandlungsberichte, v. Netherlands Ophthalmological Society

Vertikale Rlicklähmung; Nystagmus retractorius, vertikale Blick-lähmung, Konvergenzlähmung, Oberlidretraktion, Lichtareflexic der Pupillen. Ein Beitrag zur herddiagnostischen Bedeuümg der vertikalen Blicklähmung, 51

Vessels, v. Angioscotomata, Calcium dobesilate, Chorioretinal inflammatory lesions, Choroidal fluorescence, Gefässverschlüsse, Leukoencephalitis, Netzhaut-gefässschattenfigur, Newborn, Vascular occlusion

Vision binoculaire, v. Subliminal stimuli

Visual acuity, v. Acuity testing, Amyloidosis, Ectopia lentis, Netz-hautgefässschattenfigur, Skiagram

Visual field, v. Angioscotomata, Follow-up, Netzhautgefäss-schattenfigur, Retinitis pigmentosa Visually evoked response, v. Macular ERG

Vitreous body, v. Amyloidosis, Retinal detachment, Transscleral freezing, Vitreous cells

Vitreous cells; cells of the vitreous in glaucoma, 285 (V)

Wagner's vitreo-retinal degeneration,

v. Retinal detachment Wegenersche Granulomatose mit

konjunktivalem Beginn, 321 Weitwinkelglaukom, v. Cholin-

esterase

X-ray, v. Stereoscopic image amplifier

Z. Zentralnervensystem

v. Hydranence-phaly

Leukoencephalitis

Oph-thalmoplegie

Parkinson's disease

Vertikale Blicklähmung

Zerreissfestigkeit; Verlaufsunter-suchungen der Zerreissfestigkeit der Kaninchensklera nach Elektro-koagulation und Kryothermie 146 